\title{
COMPOSICIÓN QUÍMICA DE ACEITES ESENCIALES DE 10 PLANTAS AROMÁTICAS PERUANAS
}

\author{
Candy Ruiz, Camilo Díaz, Rosario Rojas*
}

\begin{abstract}
RESUMEN
Con el objetivo de encontrar aceites esenciales que puedan ser usados como atrayentes o repelentes de Thrips tabaci, una plaga que afecta al espárrago, en el presente trabajo se estudia la composición química de los aceites esenciales de 10 plantas aromáticas peruanas: Jungia paniculata (DC.) A. Gray, Piper hispidum Sw., Ambrosia arborescens Mill., Ambrosia peruviana Willd., Luma chequen (Feuillée ex Molina) A. Gray., Minthostachys setosa (Briq.) Epling, Lepechinia meyenii (Walp.) Epl., Ruta chalepensis L., Ruta graveolens L., Aristolochia silvatica Barb. Rod. Se reporta por primera vez la composición de los aceites esenciales de J. paniculata ("matico de la sierra"), A. peruviana ("marco hembra"), L. meyenii ("pachasalvia") y A. silvatica ("huampishcuna").
\end{abstract}

Palabras clave: Aceite esencial, cromatografía de gases, Perú, plantas aromáticas

\section{ABSTRACT \\ CHEMICAL COMPOSITION OF ESSENTIAL OILS FROM 10 PERUVIAN AROMATIC PLANTS}

With the aim of discovering essential oils that could be used as attractants or repellents of Thrips tabaci, an insect that affects asparagus, we report here the chemical composition of essential oils obtained from 10 Peruvian aromatic plants: Jungia paniculata (DC.) A. Gray, Piper hispidum Sw., Ambrosia arborescens Mill., Ambrosia peruviana Willd., Luma chequen (Feuillée ex Molina) A. Gray., Minthostachys setosa (Briq.) Epling, Lepechinia meyenii (Walp.) Epling, Ruta chalepensis L., Ruta graveolens L., Aristolochia silvatica Barb. Rod. Chemical composition of essential oils from J. paniculata ("Matico de la sierra"), $A$. peruviana ("Marco hembra"), L. meyenii ("Pachasalvia") and A. silvatica ("Huampishcuna") are reported here for the first time.

Key words: Aromatic plants, essential oils, gas chromatography, Perú

\section{INTRODUCCIÓN}

Los aceites esenciales son compuestos del metabolismo vegetal; la mayoría de ellos son volátiles y son responsables del aroma de las plantas. ${ }^{1}$ Dependiendo de la especie, se calcula que un aceite esencial puede contener entre 50 a 300 compuestos químicos, los cuales pertenecen a los grupos de hidrocarburos terpénicos, alcoholes, aldehídos, cetonas, éteres, ésteres, compuestos fenólicos, fenilpropanoides, entre otros. ${ }^{2}$ Las características químicas

Unidad de Investigación en Productos Naturales, Laboratorios de Investigación y Desarrollo, Facultad de Ciencias y Filosofía. Universidad Peruana Cayetano Heredia, Av. Honorio Delgado 430, Lima 31, Perú,

rosario.rojas@upch.pe 
específicas de los aceites esenciales varían en función de la zona de cultivo y condiciones ambientales. $^{3}$

Los aceites esenciales cumplen un rol ecológico como atrayentes de polinizadores y dispersores de frutos y semillas; además pueden actuar como repelentes de insectos y forman parte de la defensa química de las plantas. Tienen importancia comercial en la industria de alimentos, farmacéutica, de sabores/fragancias, cosmética y de productos de aseo. ${ }^{2}$ Asimismo, el empleo de aceites esenciales es una opción importante para el control de insectos, hongos y nemátodos, como una alternativa al uso de plaguicidas sintéticos.

Con el objetivo de encontrar aceites esenciales que puedan ser usados como atrayentes o repelentes de Thrips tabaci, una plaga que afecta al espárrago, en el presente trabajo se estudia la composición química de 10 plantas aromáticas peruanas. Los aceites esenciales obtenidos serán evaluados en campos de cultivo de espárrago con miras a conocer su potencial para el control del Thrips.

\section{PARTE EXPERIMENTAL}

Equipos. Cromatógrafo de gases Agilent Technologies 7890A acoplado a detector FID y a un detector selectivo de masas Agilent Technologies 5975.

Colecta e identificación de plantas. Las muestras vegetales fueron colectadas e identificadas por el biólogo Camilo Díaz, entre mayo a junio del 2014. Para cada una de las plantas se depositó un voucher en el herbario de la sección de Ciencias Farmacéuticas de la Universidad Peruana Cayetano Heredia.

Obtención de aceites esenciales. Cada aceite esencial fue obtenido por destilación por arrastre con vapor de agua a partir de material vegetal fresco. Una vez destilado el aceite esencial, este se separó por diferencia de densidades utilizando una probeta-florentino graduada. Luego de separar el aceite esencial de la fase acuosa, este se filtró sobre sulfato de sodio anhidro, almacenándose luego en un frasco ámbar a una temperatura de $4 \mathrm{oC}$.

Análisis por cromatografía de gases acoplada a espectrometría de masas (GC-MS). Para el análisis de cada muestra se utilizó $20 \mu \mathrm{L}$ del aceite esencial en $980 \mu \mathrm{L}$ de diclorometano, que fueron luego inyectados en el cromatógrafo de gases acoplado a un detector selectivo de masas. La separación de los compuestos en la mezcla fue llevada a cabo mediante una columna capilar apolar DB-5MS (60 m x $250 \mu \mathrm{m}$ x 0,25 $\mu \mathrm{m})(\mathrm{J} \& \mathrm{~W}$ Scientific de 5\% fenilpolimetilsiloxano).

La temperatura del inyector se mantuvo a 250 oC y la inyección se realizó en modo split (50:1). El programa de temperaturas del horno fue como sigue: temperatura inicial de $50^{\circ} \mathrm{C}$, mantenida por 5 minutos; posteriormente luego se incrementó a $10^{\circ} \mathrm{C} / \mathrm{min}$ hasta $100^{\circ} \mathrm{C}, 3$ ${ }^{\circ} \mathrm{C} /$ min hasta $150{ }^{\circ} \mathrm{C}, 7^{\circ} \mathrm{C} /$ min hasta $200^{\circ} \mathrm{C}, 1^{\circ} \mathrm{C} /$ min hasta $230{ }^{\circ} \mathrm{C}, 2^{\circ} \mathrm{C} /$ min hasta $250{ }^{\circ} \mathrm{C}$ y finalmente a $10^{\circ} \mathrm{C} / \mathrm{min}$ hasta $270{ }^{\circ} \mathrm{C}$, manteniendo la temperatura final por $1 \mathrm{~min}$. El tiempo de corrida fue de 77,8 minutos, utilizando helio como gas de arrastre a un flujo constante de $1 \mathrm{~mL} / \mathrm{min}$.

Los constituyentes de los aceites esenciales fueron identificados utilizando el software proporcionado por Agilent: MSD Chemstation (versión E02.00.493), por comparación de los espectros de masas de cada pico con los de la librería de espectros de masas de las bases de datos Flavor 2 y la del Instituto Nacional de Estándares y Tecnología (NIST, 08). 


\section{RESULTADOS Y DISCUSIÓN}

En la tabla 1 se muestra los volúmenes de aceite esencial obtenido para cada una de las 10 plantas estudiadas. El rendimiento de obtención de aceite esencial varió de 0,01 a $0,99 \%$ (Jungia paniculata y Minthostachys setosa, respectivamente).

Tabla 1. Rendimientos de obtención de aceites esenciales de 10 plantas peruanas

\begin{tabular}{|c|c|c|c|c|c|}
\hline $\begin{array}{l}\text { Nombre científico } \\
\text { (Nombre común) }\end{array}$ & $\begin{array}{c}\text { Familia } \\
\text { (No. Voucher) }\end{array}$ & $\begin{array}{c}\text { Parte de la } \\
\text { planta } \\
\text { usada }\end{array}$ & $\begin{array}{l}\text { Peso } \\
(\mathrm{kg})\end{array}$ & $\begin{array}{l}\text { Volumen Aceite } \\
\text { esencial (mL) }\end{array}$ & $\begin{array}{c}\text { Rendimiento } \\
\% \\
\text { (v/p) }\end{array}$ \\
\hline $\begin{array}{c}\text { Jungia paniculata } \\
\text { (DC.) A. Gray } \\
\text { (matico de la sierra) }\end{array}$ & $\begin{array}{c}\text { Asteraceae } \\
\text { (HEPLAME-CDS- } \\
\text { 13140-2014) }\end{array}$ & $\begin{array}{c}\text { Tallo y hoja } \\
\text { (Huaraz) }\end{array}$ & 7,3 & 1 & 0,01 \\
\hline $\begin{array}{l}\text { Piper hispidum Sw. } \\
\text { (matico hoja lisa) }\end{array}$ & $\begin{array}{c}\text { Piperaceae } \\
\text { (HEPLAME-CDS- } \\
\text { 13116-2014) }\end{array}$ & $\begin{array}{l}\text { Hojas } \\
\text { (Iquitos) }\end{array}$ & 12,2 & 23 & 0,19 \\
\hline $\begin{array}{c}\text { Ambrosia } \\
\text { arborescens Mill. } \\
\text { (marco macho) }\end{array}$ & $\begin{array}{c}\text { Asteraceae } \\
\text { (HEPLAME-CDS- } \\
\text { 13126-2014) }\end{array}$ & $\begin{array}{l}\text { Tallo y hoja } \\
\text { (Huánuco) }\end{array}$ & 21,8 & 9 & 0,04 \\
\hline $\begin{array}{c}\text { Ambrosia peruviana } \\
\text { Willd. (marco } \\
\text { hembra) }\end{array}$ & $\begin{array}{c}\text { Asteraceae } \\
\text { (HEPLAME-CDS- } \\
\text { 13125-2014) }\end{array}$ & $\begin{array}{l}\text { Tallo y hoja } \\
\text { (Huánuco) }\end{array}$ & 9,4 & 16 & 0,17 \\
\hline $\begin{array}{c}\text { Luma chequen } \\
\text { (Feuillée ex Molina) } \\
\text { A. Gray. } \\
\text { (arrayán) }\end{array}$ & $\begin{array}{c}\text { Myrtaceae } \\
\text { (HEPLAME-CDS- } \\
\text { 13128-2014) }\end{array}$ & $\begin{array}{l}\text { Tronco } \\
\text { (Huaraz) }\end{array}$ & 18,2 & 35 & 0,19 \\
\hline $\begin{array}{c}\text { Minthostachys } \\
\text { setosa (Briq.) Epling } \\
\text { (muña) }\end{array}$ & $\begin{array}{c}\text { Lamiaceae } \\
\text { (HEPLAME-CDS- } \\
\text { 13137-2014) }\end{array}$ & $\begin{array}{l}\text { Tallo y hoja } \\
\text { (Huaraz) }\end{array}$ & 19,2 & 19 & 0,99 \\
\hline $\begin{array}{l}\text { Lepechinia meyenii } \\
\text { (Walp.) Epling } \\
\text { (pachasalvia) }\end{array}$ & $\begin{array}{c}\text { Lamiaceae } \\
\text { (HEPLAME-CDS- } \\
\text { 13143-2014) }\end{array}$ & $\begin{array}{c}\text { Tallo y hoja } \\
\text { (Huaraz) }\end{array}$ & 6,5 & 20 & 0,31 \\
\hline $\begin{array}{l}\text { Ruta chalepensis L. } \\
\text { (ruda macho) }\end{array}$ & $\begin{array}{c}\text { Rutaceae } \\
\text { (HEPLAME-CDS- } \\
\text { 13142-2014) }\end{array}$ & $\begin{array}{c}\text { Tallo y hoja } \\
\text { (Huaraz) }\end{array}$ & 13,7 & 30 & 0,22 \\
\hline $\begin{array}{l}\text { Ruta graveolens L. } \\
\text { (ruda hembra) }\end{array}$ & $\begin{array}{c}\text { Rutaceae } \\
\text { (HEPLAME-CDS- } \\
\text { 13141-2014) }\end{array}$ & $\begin{array}{l}\text { Tallo y hoja } \\
\text { (Huaraz) }\end{array}$ & 9,4 & 25 & 0,27 \\
\hline $\begin{array}{c}\text { Aristolochia } \\
\text { silvatica } \text { Barb. Rod. } \\
\text { (huampishcuna) }\end{array}$ & $\begin{array}{c}\text { Aristolochiaceae } \\
\text { (HEPLAME-CDS- } \\
\text { 13114-2014) }\end{array}$ & $\begin{array}{l}\text { Troncos } \\
\text { (Iquitos) }\end{array}$ & 11,0 & 13 & 0,12 \\
\hline
\end{tabular}


En las tablas 2 a 11 se muestra las composiciones químicas relativas de los componentes de los aceites esenciales de las 10 plantas estudiadas.

En la tabla 2 se puede observar que los componentes mayoritarios del aceite esencial de Jungia paniculata son $\beta$-Cariofileno (35,91\%), D-Limoneno $(7,18 \%)$ y $\alpha$-Cariofileno $(5,82 \%)$. Casado et al. reportaron que el extracto de $J$. paniculata posee actividad antioxidante y antiinflamatoria. ${ }^{4} \mathrm{D}^{\prime}$ Agostino et al. reportaron para el extracto metanólico los glicósidos de flavonoides 3-O- $\beta$-D-glucofuranosido 3,5,7,8,4'-pentahidroxiflavona y 3-O- $\beta$-D- $(6$ "galloil)-glucopiranosido de kaempferol. ${ }^{5}$ Esta es la primera vez que se reporta la composición química del aceite esencial de Jungia paniculata.

Tabla 2. Composición del aceite esencial de Jungia paniculata ("matico de la sierra")

\begin{tabular}{lcc}
\hline \multicolumn{1}{c}{ Compuesto } & $\begin{array}{c}\mathbf{t}_{\mathbf{R}} \\
(\mathrm{min})\end{array}$ & $\begin{array}{c}\text { Abundancia } \\
\text { relativa (\%) }\end{array}$ \\
\hline$o$-Cimeno & 18,05 & 0,31 \\
D-Limoneno & 18,24 & 7,18 \\
3,7-Dimetil- 6-octenal & 22,48 & 0,42 \\
Mentona & 22,95 & 0,65 \\
Acetato de lavandulol & 30,80 & 0,51 \\
Copaeno & 31,21 & 0,84 \\
B-Cariofileno & 32,63 & 35,91 \\
$\alpha$-Bergamoteno & 32,74 & 1,83 \\
Decahidro-1,1,7-trimetil-4-metileno-1H- & 33,12 & 0,38 \\
Cicloprop[e]azuleno & 33,59 & 5,82 \\
$\alpha$-Cariofileno & 33,97 & 3,85 \\
$\alpha$-Curcumeno & 34,16 & 0,39 \\
$\beta$-Farneseno & 35,02 & 0,79 \\
N.I. $\left(\mathrm{C}_{15} \mathrm{H}_{26} \mathrm{O}\right)$ & 36,16 & 1,17 \\
N.I. $\left(\mathrm{C}_{15} \mathrm{H}_{24} \mathrm{O}\right)$ & 37,09 & 36,49 \\
Óxido de cariofileno & 37,83 & 2,86 \\
N.I. $\left(\mathrm{C}_{15} \mathrm{H}_{24} \mathrm{O}\right)$ & 39,45 & 0,60 \\
N.I. $\left(\mathrm{C}_{15} \mathrm{H}_{24} \mathrm{O}\right)$ & & \\
\hline
\end{tabular}

N.I.: No identificado

En la tabla 3 se resume los componentes del aceite esencial de Piper hispidum. Los componentes mayoritarios son $\alpha$-Felandreno (22,30\%), Eucaliptol $(15,49 \%)$ y $\alpha$-Pineno $(14,82 \%)$. Pino et al. estudiaron el aceite esencial de P. hispidum proveniente de Cuba, reportando para él diferentes componentes mayoritarios: $\beta$-Eudesmol $(17,5 \%)$ y trans-6vinil-4,5,6,7-tetrahidro-3,6-dimetil-5-isopropenilbenzofurano $(12,9 \%){ }^{6}$ 
Tabla 3. Composición del aceite esencial de Piper hispidum ("matico hoja lisa")

\begin{tabular}{|c|c|c|}
\hline Compuesto & $\begin{array}{c}\mathbf{t}_{\mathbf{R}} \\
(\min )\end{array}$ & $\begin{array}{l}\text { Abundancia } \\
\text { relativa (\%) }\end{array}$ \\
\hline$\alpha$-Pineno & 15,14 & 14,82 \\
\hline ß-Pineno & 16,60 & 6,40 \\
\hline ß-Mirceno & 16,66 & 1,19 \\
\hline$\alpha$-Felandreno & 17,44 & 22,30 \\
\hline 3-Careno & 17,53 & 2,84 \\
\hline$\alpha$-Terpineno & 17,77 & 0,70 \\
\hline$o$-Cimeno & 18,03 & 0,95 \\
\hline D-Limoneno & 18,21 & 8,77 \\
\hline ß-Felandreno & 18,33 & 1,47 \\
\hline Eucaliptol & 18,39 & 15,49 \\
\hline$\gamma$-Terpineno & 19,15 & 1,19 \\
\hline Copaeno & 31,18 & 0,84 \\
\hline ß-Elemeno & 31,50 & 1,05 \\
\hline ß-Cariofileno & 32,58 & 2,43 \\
\hline Allo-aromadendreno & 33,09 & 0,61 \\
\hline$\alpha$-cariofileno & 33,56 & 0,48 \\
\hline Germacreno D & 34,19 & 0,91 \\
\hline Eremofileno & 34,44 & 1,22 \\
\hline Biciclogermacreno & 34,57 & 2,79 \\
\hline$\delta$-Cadineno & 35,02 & 0,65 \\
\hline N.I. $\left(\mathrm{C}_{13} \mathrm{H}_{14} \mathrm{O}_{3}\right)$ & 39,31 & 12,90 \\
\hline
\end{tabular}

N.I.: No identificado

En la tabla 4 se observa que los componentes del aceite esencial de Ambrosia arborescens son Germacreno D (36,96\%) y $\beta$-Himachaleno (30,62\%). De Leo et al. estudiaron el extracto de $A$. arborescens y reportaron diferentes sesquiterpenos y diterpenos entre los cuales destacan:

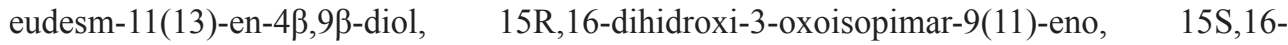
dihidroxi-3-oxoisopimar-9(11)-eno, $1 \alpha$-hidroxi-7-oxo-iso-anhidrooplopanona, $10 \alpha$-hidroxi11,13-dihidro-5-epi-psilostachyin y 4-O- $\beta$-d-glucopiranósido de 4 $\beta$-hidroxipseudoguaian12,6-ólido, además de otros 12 sesquiterpenos. ${ }^{7}$ 
Figura 4. Composición del aceite esencial de Ambrosia arborescens ("marco macho")

\begin{tabular}{|c|c|c|}
\hline Compuesto & $\begin{array}{ll}\mathbf{t}_{\mathbf{R}} & (\mathrm{min})\end{array}$ & Abundancia relativa (\%) \\
\hline Santolina trieno & 13,95 & 0,50 \\
\hline Origaneno & 14,82 & 0,76 \\
\hline$\alpha$-Pineno & 15,13 & 0,69 \\
\hline Sabineno & 16,34 & 4,87 \\
\hline B-Pineno & 16,60 & 0,72 \\
\hline B-Mirceno & 16,66 & 1,52 \\
\hline 1,6-Dimetilhepta-1,3,5-trieno & 16,77 & 0,76 \\
\hline$\alpha$-Felandreno & 17,43 & 1,09 \\
\hline$\alpha$-Terpineno & 17,77 & 0,47 \\
\hline D-Limoneno & 18,20 & 1,23 \\
\hline$\gamma$-Terpineno & 19,16 & 0,88 \\
\hline$\alpha$-Terpinoleno & 20,18 & 0,71 \\
\hline N.I. $\left(\mathrm{C}_{10} \mathrm{H}_{14} \mathrm{O}\right)$ & 20,76 & 0,70 \\
\hline 5-(1,1-dimetiletil)-1,3-ciclopentadieno & 21,60 & 1,63 \\
\hline Copaeno & 31,19 & 0,55 \\
\hline ß-Elemeno & 31,50 & 1,18 \\
\hline B-Cariofileno & 32,59 & 2,42 \\
\hline$\alpha$-Cariofileno & 33,56 & 0,71 \\
\hline ß-Himachaleno & 33,92 & 30,62 \\
\hline$\alpha$-Curcumeno & 33,97 & 2,31 \\
\hline Germacreno D & 34,24 & 36,96 \\
\hline$\alpha$-Farneseno & 34,40 & 1,62 \\
\hline B-Selineno & 34,47 & 2,12 \\
\hline$\alpha$-Selineno & 34,62 & 2,29 \\
\hline$\alpha$-Amorfeno & 34,99 & 0,23 \\
\hline$\delta$-Cadineno & 35,03 & 1,38 \\
\hline$\alpha$-Gurjuneno & 35,48 & 0,81 \\
\hline N.I. $\left(\mathrm{C}_{15} \mathrm{H}_{24} \mathrm{O}\right)$ & 37,51 & 0,28 \\
\hline
\end{tabular}


En la tabla 5 se observa que los componentes del aceite esencial de Ambrosia peruviana son Germacreno D (32,66\%), ß-Himachaleno (16,78\%), acetato de bornilo $(10,97 \%)$ y Biciclogermacreno (10,20\%). Aponte et al. reportan la presencia de diversos sesquiterpenoides en el extracto etanólico de las hojas de $A$. peruviana ${ }^{8}$ Esta es la primera vez que se reporta la composición química de su aceite esencial, el cual es algo similar a la del aceite esencial de $A$. arborescens.

Figura 5. Composición del aceite esencial de Ambrosia peruviana ("marco hembra")

\begin{tabular}{|c|c|c|}
\hline Compuesto & $\begin{array}{c}\mathbf{t}_{\mathbf{R}} \\
(\mathrm{min})\end{array}$ & $\begin{array}{l}\text { Abundancia } \\
\text { relativa (\%) }\end{array}$ \\
\hline$\alpha-$ Pineno & 15,14 & 2,27 \\
\hline Canfeno & 15,73 & 2,07 \\
\hline Sabineno & 16,35 & 0,64 \\
\hline ß-Pineno & 16,62 & 7,39 \\
\hline N.I. $\left(\mathrm{C}_{9} \mathrm{H}_{14}\right)$ & 16,77 & 1,13 \\
\hline ß-felandreno & 18,34 & 1,17 \\
\hline 1,2,5,5-tetrametil-1,3-ciclopentadieno & 21,01 & 0,69 \\
\hline 1,6-Dimetilhepta-1,3,5-trieno & 21,61 & 2,79 \\
\hline Alcanfor & 22,76 & 0,90 \\
\hline Acetato de bornilo & 27,95 & 10,97 \\
\hline Copaeno & 31,20 & 0,60 \\
\hline ß-Cubebeno & 31,51 & 1,66 \\
\hline B-Cariofileno & 32,60 & 2,56 \\
\hline ß-Farneseno & 33,06 & 0,41 \\
\hline$\alpha$-Cariofileno & 33,57 & 1,04 \\
\hline ß-Himachaleno & 33,91 & 16,78 \\
\hline Curcumeno & 33,96 & 1,67 \\
\hline N.I. $\left(\mathrm{C}_{15} \mathrm{H}_{24} \mathrm{O}_{2}\right)$ & 34,04 & 0,50 \\
\hline Germacreno D & 34,25 & 32,66 \\
\hline Biciclogermacreno & 34,61 & 10,20 \\
\hline$\delta$-Cadineno & 35,04 & 0,64 \\
\hline Carotol & 37,58 & 0,84 \\
\hline N.I. $\left(\mathrm{C}_{12} \mathrm{H}_{18} \mathrm{O}_{2}\right)$ & 38.11 & 0.42 \\
\hline
\end{tabular}

N.I.: No identificado 
En la tabla 6 se observa que los principales componentes del aceite esencial de Luma chequen son D Limoneno (49,71\%) y Eucaliptol $(13,79 \%)$. Vallverdú et al. encontraron que los compuestos mayoritarios fueron $\alpha$-pineno $(57,3 \%)$ y eucaliptol $(7,5 \%) .9$ Mientras que Gonçalves et al. encontraron como componentes mayoritarios a $\alpha$-pineno $(57,1 \%)$, eucaliptol $(12,1 \%)$ y linalool $(5,5 \%) .^{10}$

Figura 6. Composición del aceite esencial de Luma chequen ("arrayán")

\begin{tabular}{|c|c|c|}
\hline Compuesto & $\underset{(\min )}{\mathbf{t}_{\mathbf{R}}}$ & $\begin{array}{l}\text { Abundancia } \\
\text { relativa (\%) }\end{array}$ \\
\hline Origaneno & 14,84 & 0,49 \\
\hline$\alpha$-Pineno & 15,16 & 7,32 \\
\hline Sabineno & 16,36 & 0,44 \\
\hline B-Pineno & 16,62 & 1,60 \\
\hline$\alpha$-Felandreno & 17,46 & 0,52 \\
\hline$p$-Cimeno & 18,07 & 3,15 \\
\hline D-Limoneno & 18,28 & 49,71 \\
\hline Eucaliptol & 18,44 & 13,79 \\
\hline$\gamma$-Terpineno & 19,19 & 1,77 \\
\hline Acetato de mirtenilo & 29,27 & 0,56 \\
\hline ß-Elemeno & 31,53 & 1,41 \\
\hline B-Cariofileno & 32,61 & 2,58 \\
\hline Aromadendreno & 33,12 & 0,61 \\
\hline$\alpha$-Cariofileno & 33,58 & 0,57 \\
\hline Allo-aromadendreno & 33,71 & 0,48 \\
\hline$\alpha$-Gurjuneno & 33,94 & 0,80 \\
\hline B-Selineno & 34,48 & 3,72 \\
\hline $\begin{array}{l}\text { 2-Isopropenil-4a,8-dimetil-1,2,3,4,4 } \alpha, 5,6,7 \text { - } \\
\text { octahidronaftaleno }\end{array}$ & 34,64 & 5,34 \\
\hline$\gamma$-Cadineno & 35,00 & 0,55 \\
\hline$\delta$-Cadineno & 35,05 & 0,76 \\
\hline Nerolidol & 36,06 & 1,84 \\
\hline Óxido de cariofileno & 37,05 & 2,00 \\
\hline
\end{tabular}

N.I.: No identificado 
En la tabla 7 se observa que los componentes mayoritarios del aceite esencial de Minthostachys setosa son Timol (22,30\%), D-Carvona (13,01\%) y Mentona (9,18\%). Senatore F. obtuvo aceite esencial de $M$. setosa con un rendimiento de $0,68 \%$; el componente mayoritario fue el monoterpeno pulegona. ${ }^{11}$

Figura 7. Composición del aceite esencial de Minthostachys setosa ("muña")

\begin{tabular}{|c|c|c|}
\hline Compuesto & $\mathbf{t}_{\mathbf{R}} \quad(\min )$ & $\begin{array}{l}\text { Abundancia } \\
\text { relativa (\%) }\end{array}$ \\
\hline Origaneno & 15,24 & 1,55 \\
\hline$\alpha$-Pineno & 15,53 & 0,63 \\
\hline Sabineno & 16,57 & 0,42 \\
\hline$\beta$-Mirceno & 16,83 & 1,61 \\
\hline$\alpha$-Terpineno & 17,77 & 0,63 \\
\hline$o$-Cimeno & 17,97 & 4,54 \\
\hline trans-Ocimeno & 18,06 & 0,66 \\
\hline D-Limoneno & 18,12 & 3,63 \\
\hline cis-Ocimeno & 18,38 & 1,39 \\
\hline$\gamma$-Terpineno & 18,87 & 2,90 \\
\hline Mentona & 21,54 & 9,18 \\
\hline D-Isomentona & 21,76 & 1,96 \\
\hline$d$-Dihidrocarvona & 22,47 & 5,38 \\
\hline Carvona & 22,63 & 0,43 \\
\hline Pulegona & 23,32 & 2,44 \\
\hline D-Carvona & 23,42 & 13,01 \\
\hline N.I. $\left(\mathrm{C}_{10} \mathrm{H}_{16} \mathrm{O}_{2}\right)$ & 23,60 & 6,07 \\
\hline Piperitona & 23,66 & 5,49 \\
\hline Carvacrol & 24,33 & 6,56 \\
\hline Acetato de timol & 25,17 & 0,75 \\
\hline Eucarvona & 25,30 & 0,78 \\
\hline Timol & 25,56 & 22,30 \\
\hline N.I. $\left(\mathrm{C}_{10} \mathrm{H}_{14} \mathrm{O}_{2}\right)$ & 25,64 & 1,59 \\
\hline$\beta$-Cariofileno & 26,87 & 2,89 \\
\hline$\alpha$ - Cariofileno & 27,48 & 0,44 \\
\hline Germacreno D & 27,88 & 1,96 \\
\hline Biciclogermacreno & 28,12 & 0,81 \\
\hline
\end{tabular}

N.I.: No identificado 
En la tabla 8 se observa que los componentes principales del aceite esencial de Lepechinia meyenii son $\alpha$-Pineno (29,87\%), Eucaliptol $(13,25 \%)$ y $\beta$-Pineno $(9,64 \%)$. Chirinos et al. encontraron que el extracto de L. meyenii posee buena actividad antioxidante. ${ }^{12}$ Esta es la primera vez que se reporta la composición química de su aceite esencial.

Figura 8. Composición del aceite esencial de Lepechinia meyenii ("pachasalvia")

\begin{tabular}{|c|c|c|}
\hline Compuesto & $\begin{array}{c}\mathbf{t}_{\mathbf{R}} \\
(\mathrm{min})\end{array}$ & $\begin{array}{l}\text { Abundancia } \\
\text { relativa (\%) }\end{array}$ \\
\hline$\alpha$-Pineno & 15,17 & 29,87 \\
\hline Canfeno & 15,73 & 0,36 \\
\hline B-Pineno & 16,62 & 9,64 \\
\hline ß-Mirceno & 16,68 & 1,77 \\
\hline$\alpha$-felandreno & 17,45 & 0,49 \\
\hline 3-Careno & 17,56 & 9,89 \\
\hline$\alpha$-Terpineno & 17,79 & 0,57 \\
\hline$p$-Cimeno & 18,05 & 0,51 \\
\hline D-Limoneno & 18,24 & 9,77 \\
\hline B-felandreno & 18,36 & 2,32 \\
\hline Eucaliptol & 18,41 & 13,25 \\
\hline$\gamma$-Terpineno & 19,18 & 0,94 \\
\hline 4-Careno & 20,20 & 0,85 \\
\hline N.I. $\left(\mathrm{C}_{12} \mathrm{H}_{18} \mathrm{O}_{2}\right)$ & 28,30 & 0,63 \\
\hline Acetato de mirtenilo & 29,26 & 0,38 \\
\hline Copaeno & 31,20 & 0,82 \\
\hline$\alpha$-Gurjuneno & 32,20 & 0,71 \\
\hline B- Cariofileno & 32,61 & 4,44 \\
\hline Aromadendreno & 33,11 & 2,14 \\
\hline$\alpha$-Cariofileno & 33,58 & 0,77 \\
\hline Curcumeno & 33,96 & 0,51 \\
\hline$\alpha$-Farneseno & 34,02 & 0,60 \\
\hline Varidifloreno & 34,45 & 1,17 \\
\hline$\alpha$-Amorfeno & 35,00 & 0,79 \\
\hline$\delta$-Cadineno & 35,05 & 1,97 \\
\hline Guaiol & 37,41 & 4,82 \\
\hline
\end{tabular}

N.I.: No identificado 
En la tabla 9 se observa que los componentes en mayor abundancia en el aceite esencial de Ruta chalepensis son 2-Undecanona $(58,16 \%)$ y 2 -Nonanona $(25,26 \%)$. Por otro lado, el aceite esencial de Ruta graveolens también posee los mismos compuestos mayoritarios: Undecanona $(40,88 \%)$ y 2 -Nonanona $(28,96 \%)$ (Tabla 10$)$. Ferhat et al. reportaron que los aceites esenciales de Ruta chalepensis, Ruta montana y Ruta graveolens contienen 2-Undecanona (1,79-84,2\%), 2-decanona (0,1-11,6\%), 2-nonanona (5,2-33,6\%) y 2-nonanilacetato $(2.8-20,9 \%){ }^{13}$

Figura 9. Composición del aceite esencial de Ruta chalepensis ("ruda macho")

\begin{tabular}{lcc}
\hline \multicolumn{1}{c}{ Compuesto } & $\begin{array}{c}\mathbf{t}_{\mathbf{R}} \\
(\mathrm{min})\end{array}$ & $\begin{array}{c}\text { Abundancia } \\
\text { relativa (\%) }\end{array}$ \\
\hline 2-Nonanona & 20,14 & 25,26 \\
5,6-dietenil-1-metil ciclohexeno & 22,44 & 3,65 \\
2-Decanona & 23,92 & 1,47 \\
N.I. $\left(\mathrm{C}_{13} \mathrm{H}_{24} \mathrm{O}_{2}\right)$ & 25,43 & 7,47 \\
2-Undecanona & 28,01 & 58,16 \\
N.I. $\left(\mathrm{C}_{12} \mathrm{H}_{24} \mathrm{O}\right)$ & 30,41 & 1,03 \\
2-Dodecanona & 31,33 & 0,64 \\
N.I. $\left(\mathrm{C}_{16} \mathrm{H}_{32} \mathrm{O}_{2}\right)$ & 32,26 & 1,50 \\
2-Tridecanona & 34,14 & 0,82 \\
\hline
\end{tabular}

N.I.: No identificado

Figura 10. Composición del aceite esencial de Ruta graveolens ("ruda hembra")

\begin{tabular}{lcc}
\hline \multicolumn{1}{c}{ COMPUESTO } & $\begin{array}{c}\mathbf{t}_{\mathbf{R}} \\
(\mathrm{min})\end{array}$ & $\begin{array}{c}\text { ABUNDANCIA } \\
\text { RELATIVA } \\
(\%)\end{array}$ \\
\hline 2-Nonanona & 20,13 & 28,96 \\
Acetato de 2-Octilo & 21,61 & 0,39 \\
N.I. $\left(\mathrm{C}_{11} \mathrm{H}_{16}\right)$ & 22,13 & 0,43 \\
5,6-dietenil-1-metil-ciclohexeno & 22,43 & 4,22 \\
2-Decanona & 23,91 & 1,19 \\
N.I. $\left(\mathrm{C}_{13} \mathrm{H}_{24} \mathrm{O}_{2}\right)$ & 25,43 & 16,29 \\
2-Undecanona & 27,98 & 40,88 \\
2-Metil undecanal & 30,40 & 0,87 \\
2-Dodecanona & 31,32 & 0,49 \\
N.I. $\left(\mathrm{C}_{16} \mathrm{H}_{32} \mathrm{O}_{2}\right)$ & 32,25 & 1,94 \\
B-Cariofileno & 32,58 & 3,40 \\
a-Cariofileno & 33,56 & 0,37 \\
2-Tridecanona & 34,13 & 0,57 \\
\hline
\end{tabular}

N.I.: No identificado 
En la tabla 11 se observa que los componentes del aceite esencial de Aristolochia silvatica son $\beta$-Cariofileno (13,75\%), $\alpha$-Pineno $(11,12 \%)$ y Dihidro aromadendreno $(9,96 \%)$. Esta es la primera vez que se reporta la composición química del aceite esencial de A. silvatica.

Figura 11. Composición del aceite esencial de Aristolochia silvatica ("huampishcuna")

\begin{tabular}{|c|c|c|}
\hline Compuesto & $\mathbf{t}_{\mathbf{R}} \quad(\min )$ & $\begin{array}{l}\text { Abundancia } \\
\text { relativa (\%) }\end{array}$ \\
\hline$\alpha-$ Pineno & 15,21 & 11,12 \\
\hline Canfeno & 15,78 & 2,23 \\
\hline ß-Pineno & 16,67 & 2,64 \\
\hline D-Limoneno & 18,28 & 0,55 \\
\hline Acetato de bornilo & 28,00 & 1,15 \\
\hline Copaeno & 31,26 & 1,43 \\
\hline ß-Elemeno & 31,57 & 1,29 \\
\hline$\alpha$-Gurjuneno & 32,25 & 3,41 \\
\hline$\beta$-Cariofileno & 32,66 & 13,75 \\
\hline N.I. $\left(\mathrm{C}_{15} \mathrm{H}_{22}\right)$ & 32,87 & 0,63 \\
\hline B-Gurjuneno & 32,99 & 6,36 \\
\hline Dihidro aromadendreno & 33,16 & 9,96 \\
\hline$\alpha$-Muuroleno & 33,28 & 0,78 \\
\hline N.I. $\left(\mathrm{C}_{14} \mathrm{H}_{20}\right)$ & 33,52 & 0,67 \\
\hline$\alpha$-Cariofileno & 33,62 & 1,96 \\
\hline N.I. $\left(\mathrm{C}_{15} \mathrm{H}_{24}\right)$ & 33,85 & 0,57 \\
\hline$\alpha$-Longipineno & 33,93 & 0,74 \\
\hline Curcumeno & 34,01 & 2,50 \\
\hline Germacreno D & 34,26 & 1,62 \\
\hline Zingibereno & 34,35 & 2,22 \\
\hline$\gamma$-Muuroleno & 34,59 & 1,01 \\
\hline Biciclogermacreno & 34,64 & 1,87 \\
\hline Di-epi- $\alpha$-cedreno & 34,74 & 1,77 \\
\hline$\alpha$-Amorfeno & 35,05 & 0,97 \\
\hline$\delta$-Cadineno & 35,10 & 5,04 \\
\hline ß-Sesquifelandreno & 35,16 & 1,00 \\
\hline Elemol & 36,08 & 6,21 \\
\hline Oxido de cariofileno & 37,10 & 1,05 \\
\hline N.I. $\left(\mathrm{C}_{15} \mathrm{H}_{22} \mathrm{O}\right)$ & 38,25 & 1,41 \\
\hline$\alpha$-Elemeno & 38,67 & 5,31 \\
\hline N.I. $\left(\mathrm{C}_{15} \mathrm{H}_{26} \mathrm{O}\right)$ & 39,28 & 5,07 \\
\hline N.I. $\left(\mathrm{C}_{15} \mathrm{H}_{26} \mathrm{O}\right)$ & 39,36 & 3,71 \\
\hline
\end{tabular}

N.I.: No identificado 


\section{CONCLUSIONES}

En el presente estudio se determinó la composición química de 10 aceites esenciales de plantas peruanas por medio de cromatografía de gases acoplada a espectrometría de masas. Se reporta, por primera vez, la composición de los aceites esenciales de Jungia paniculata ("matico de la sierra"), Ambrosia peruviana ("marco hembra"), Lepechinia meyenii ("pachasalvia") y Aristolochia silvatica ("huampishcuna").

\section{AGRADECIMIENTOS}

El presente trabajo de investigación fue financiado por el Fondo de Investigación y Desarrollo para la Competitividad - FIDECOM (Contrato No. 057-FINCyT-FIDECOM-PIPEA-2013).

\section{BIBLIOGRAFÍA}

1. Moreno, S., Crescente, O., Ortiz, S., Quintero, M. Composición química y actividad tóxica del aceite esencial de Simsia pubescens Triana. Rev. Ciencia y Tecnol. Amer. 2006; 31: 145-147

2. Stashenko, Elena E. Aceites esenciales. UIS-CENIVAM. Bucaramanga, Colombia 2009. 180 pp.

3. Collura, A. Variaciones del rendimiento y composición química de las especies aromáticas y medicinales. Bol. Soc. Arg. Invest. Prod. Arom. 1985; 4: 6-15.

4. Casado, R., Landa, A., Calvo, J.J., Del Carmen Terencio, M., Calvo, M.I. Antiinflammatory and antioxidant activities of Jungia paniculata. Pharm. Biol. 2010; 48: 897-905.

5. D'Agostino, M., Senatore, F., De Feo, V., De Simone, F. Flavonol glycosides from Jungia paniculata. Fitoterapia 1995; 66: 283-284.

6. Pino, J.A, Marbot, R., Bello, A., Urquiola, A. Composition of the essential oil of Piper hispidum Sw. from Cuba J. Essent.Oil Res. 2004; 16: 459-460.

7. De Leo, M., Saltos, M.B.V., Puente, B.F.N., De Tommasi, N., Braca, A. Sesquiterpenes and diterpenes from Ambrosia arborescens. Phytochemistry. 2010; 71: 804-809.

8. Aponte, J.C., Yang, H., Vaisberg, A.J., Castillo, D., Málaga, E., Verástegui, M., Casson, L.K., Stivers, N., Bates, P.J., Rojas, R., Fernández, I., Lewis, W.H., Sarasara, C., Sauvain, M., Gilman, R.H., Hammond, G.B. Cytotoxic and anti-infective sesquiterpenes present in Plagiochila disticha (Plagiochilaceae) and Ambrosia peruviana (Asteraceae). Planta Med. 2010; 76: 705-707.

9. Vallverdú, C., Vila, R., Tomi, F., Carhuapoma, M., Casanova, J., Cañigueral, S. Composition of the essential oil from leaves and twigs of Luma chequen. Flav. Fragr. J. 2006; 21: 241-243.

10. Gonçalves, M.J., Cavaleiro, C., Da Cunha, A.P., Salgueiro, L.R. Chemical composition and antimicrobial activity of the commercially available oil of Luma chequen (Molina) A. Gray. Flav. Fragr. J. 2006; 21: 241-243.

11. Senatore F. Volatile constituents of Minthostachys setosa (Briq.) Epl. (Lamiaceae) from Peru. Flav. Fragr. J. 1998; 4: 263-265.

12. Chirinos, R., Pedreschi, R., Rogez, H., Larondelle, Y., Campos, D. Phenolic compound contents and antioxidant activity in plants with nutritional and/or medicinal properties from the Peruvian Andean region. Ind. Crop Prod. 2013; 5: 735-738. 
13. Ferhat, M., Kabouche, A., Kabouche, Z. Comparative compositions of essential oils of three Ruta species growing in different soils. J. Mater. Environ. Sci. 2013; 47: 145-152. 